\title{
Acceptance of Children with Special Needs in Early Childhood Inclusive Education Programs
}

\section{Lathiffida Noor Jaswandi ${ }^{a}$ and Farida Kurniawati ${ }^{\mathrm{b}}$}

${ }^{a}$ Department of Early Childhood Psychology, Faculty of Psychology, Universitas Indonesia, Depok, Indonesia; ${ }^{b}$ Department of Educational Psychology, Faculty of Psychology, Universitas Indonesia, Depok, Indonesia

*Corrresponding Authors:

Farida Kurniawati

Department of Educational Psychology

Faculty of Psychology, Universitas Indonesia

J1. Lkr. Kampus Raya, Depok, Jawa Barat

Indonesia, 16424

Tel.: +62 217270004

Email address: farida1@ui.ac.id / fafadana@gmail.com 


\title{
Acceptance of Children with Special Needs in Early Childhood Inclusive Education Programs
}

\begin{abstract}
Across Indonesia, in recent times, many early childhood education institutions have embraced inclusive policies in education. Inclusive education systems encourage the acceptance of children with special needs. The sense of being accepted enables children with special needs to feel like they are not different from other children. To evaluate the benefits of the inclusive system, this study was conducted to determine levels of acceptance in early childhood for children with special needs within an inclusive kindergarten setting that boasts a high level of acceptance. The respondents were typically developing children aged 60-72 months who attend the kindergarten. The Acceptance Scale for Kindergarten-Revised (ASK-R) instrument was used to measure peer acceptance of children with special needs. Data were collected by administering the questionnaire to three respondents at a time. Quantitative descriptive statistics derived from the total ASK-R score were analyzed according to the gender and age of respondents. The results of this study suggest a moderate level of peer acceptance for children with special needs. No significant difference was found between the acceptance levels of boys and girls.
\end{abstract}

Keywords: inclusive education, early childhood, inclusive preschool, peer acceptance, student with disability

\section{Introduction}

Data from the Indonesian Central Bureau of Statistics show that 1.6 million Indonesian children have special needs. According to data from the Ministry of Education and Culture, it is estimated that $21.46 \%$ of the country's 31 million $0-6$-year-olds have special needs. Government efforts to facilitate equal education rights for all citizens entail the development of inclusive education in all formal education units. As described in Government Regulation Number 17 of 2010, this encompasses early childhood education, basic education, and secondary education units. Inclusive education, as described in Permendikbud No. 70 Tahun 2009, is an educational system that provides opportunities for all learners with disabilities, regardless of intellectual potential or talent, to attain an education in the same environment as other learners. The aim is to enable all students with special needs to gain quality education according to their needs and abilities.

Inclusive education in early childhood settings has only recently been implemented in Indonesia. It derives from the government regulations Perpres No. 60 Tahun 2013, which concerns the development of integrated holistic early childhood education, and the Ministry of Education and Culture Number 146 of 2014, based on the 2013 curriculum, which sets out curriculum guidelines for children with special needs to be developed in accordance with each individual child's potential and needs. It is important that inclusive education in early childhood education settings in Indonesia is in line with the fundamental function of early childhood education, where the education program is developed according to the needs of the child and in order to provide the correct stimulus to optimize the development of the child. In 
addition, the benefits of inclusive education in early childhood education settings support the emergence of acceptance of children with special needs in the form of positive attitudes towards such children (Nikolaraizi, Kumar, Favazza, Sideridis, Koulousiou, \& Riall, 2005).

Acceptance by peers in early childhood is measured by the extent to which the child is socially integrated and the degree to which the child is accepted and liked by peers (Beazidou \& Botsoglou, 2016). In inclusive education settings, peer acceptance of children with special needs can arise when children with special needs have the opportunity to interact with typical children (de Boer, Pijl, \& Minnaert, 2012). This closely mirrors the social development of preschool age children. At this age, children develop the ability to interact socially and to regulate their feelings (Morrison, 2009). The development of positive social abilities at an early age can be enhanced by providing opportunities to engage in activities that support interaction with other children (Morrison, 2009).

In early childhood, there is a difference in the level of peer acceptance of children with special needs. Differences between preschool boys and girls have been observed with regard to peer acceptance of children with special needs, with girls scoring higher acceptance rates than boys (de Boer, Pijl, \& Minnaert, 2012). In addition to gender, age is positively related to feelings for children with special needs (Hong, Kwon, \& Jeon, 2014). Research has also found that children with special needs are more often excluded from playing with their peers (Odom, Zercher, Marquart, Sandall, \& Brown, 2006). Early years children have been found to prefer playing with children who do not have special needs rather than those with special needs (Diamond \& Hong, 2010). This situation occurs in preschool settings in Indonesia and is seen by the early childhood education institutions as an obstacle to integration. Interviews conducted with several early childhood education professionals suggest that many children with special needs continue to experience difficulties. These difficulties are linked to building interactions between children with special needs and typical children. In these interviews, it was suggested that some children did not want to involve children with special needs in learning or play activities.

Research was conducted to explore the challenges of implementing inclusive education in early childhood education in Indonesia. The present study aimed to acquire an overview of the levels of acceptance of children with special needs in early childhood education settings where inclusive education is provided. The level of acceptance described in this research relates to the provision of optimal stimulation to generate peer acceptance in an early childhood education setting. The resulting acceptance is expected to remove some of the obstacles faced by children with special needs with regard to developing friendships and learning within school environments. The research is a baseline study to provide intervention recommendations to improve peer acceptance of children with special needs. 


\section{Methods}

\section{Participants}

Research was conducted with 110 students. Following the removal of five sets of damaged respondent data, data collected from 105 respondents were analyzed. All data were obtained with informed parental consent. Respondents consisted of 52 male students and 53 female students, ranging in age from 60 to 72 months (Mean $=67.72$ and SD = 4.925). The mean was used to describe the age of respondents to obtain detailed information related to respondents' age range.

All respondents were students without special needs who attend Inclusive Kindergarten. A student without special needs is characterized as someone who is able to interact, understand, and follow instructions. Respondent selection was conducted using the accidental sampling method, where the researchers recruited participants based on the ease of attaining the sample. Respondents were selected based on their availability and desire to participate (Gravetter \& Forzano, 2016). In the recruitment process, students became respondents following several stages of consent. Informed consent was first obtained from each child's parent or guardian. Those students who were granted consent were registered by the researchers and the teacher and jointly approached by both. The researchers, then, explained to the student the tasks he/she would do with the researcher as part of the research. Following this explanation, the researchers asked if the student was willing to help. Students who expressed unwillingness or looked uncomfortable were offered the opportunity to participate in the company of the teacher or not participate at all.

\section{Measures}

The questionnaire instrument given to respondents contained a scale of peer acceptance in early childhood, the Revised Acceptance Scale for Kindergarten (ASK-R) (Favazza \& Odom, 1996; Favazza, Philipsen, \& Kumar, 2000). ASK-R has been revised and adapted from a previous measuring instrument-ASK - that was devised specifically for kindergarten children by Paddy C. Favazza and Samuel L. Odom. The revised version, ASK-R, contains improvements on administrative implementation procedures, including barriers on questions related to disability to respondents, avoiding too many respondents in one administration, and taking note of non-conducive conditions. ASK-R adds an explanation of disability for respondents, requires the questionnaire to be administered to smaller groups of respondents, and requires the research to be conducted in a separate room in order to minimize distraction during the data collection process (Favazza, Philipsen, \& Kumar, 2000).

ASK-R consists of 18 questions that measure attitudes in preschool children, reflected in their affections and behaviors (Yu, Ostrosky, \& Fowler, 2012). It has three response scales to describe the level of acceptance in early childhood. For example, the question "Will you play with children who cannot walk?" could be answered by selecting from a response scale of symbols representing emotional expression: YES (Smile), NO (Sad), and DON'T KNOW (Thinking). 
Researchers translated the ASK-R into Indonesian and adapted it to Indonesian cultural conditions. The Indonesian version of the ASK-R was then translated back into English. Having determined that the Indonesian translation had the same meaning as the original measuring instrument, researchers judged the suitability of the new measuring instrument for a pilot study. The pilot study was conducted with 34 children, aged 5-6 years, in a kindergarten group. The results of the pilot study obtained a Cronbach's Alpha score of 0.809 and the range of values for corrected item-total correlation was $0.007-0.767$. The results of the pilot study showed four items with a corrected item-total correlation value of $<0.3$. These four items were, therefore, deleted, and with the remaining 14 items, a Cronbach's Alpha of 0.863 was obtained, and the range of values for corrected item-total correlation was 0.230-0.884. Based on the value of Cronbach's Alpha and corrected item-total correlation, it was concluded that the 14-question Indonesian version of ASK-R could be administered to kindergarten children in Indonesia.

\section{Procedure}

The questionnaire was administered and data was collected from three children at a time. Each questionnaire session began with the researchers explaining disability by sharing materials with the children. The materials consisted of individual sheets of paper, each of which contained a disability-related word and pictures of examples of physically disabled children. These images included physical disability (hearing impairment), visual impairment (blindness), hearing disability (hearing impairment), and disability in speaking (speech impairment). The disability-related material was explained through discussions with the respondents; the researchers posed questions regarding what the children already knew about disability and what they knew about the images in the pictures provided. Following this, discussion then continued about the extent of the respondents' understanding of differences and similarities. This required the respondents to answer questions about ways in which they are different from or similar to their friends. Upon conclusion of these discussions, the researchers explained to the children how the questionnaire would work.

The researchers explained the three choices (yes, no, don't know) and their corresponding symbols (smile, sadness, thinking) on the response sheet. Each respondent was then given a stamp to mark the choice in the response sheet that matched the respondent's feelings. The researchers distributed an exercise response sheet to each of the three children and read a series of exercise questions in order to give respondents the opportunity to understand how data collection would work. Once the researchers were satisfied that all respondents could follow the instructions, the questionnaire response sheet was distributed, and respondents were instructed to complete it according to the same instructions they had been given in the previous exercise. The researchers then read out the questions in the measuring instrument. Respondents were given time to choose a response appropriate to their feelings and to register that response by stamping one of the expression images. Data collection took 20-30 minutes for each set of three respondents. 


\section{Data Analysis}

The quantitative data resulting from the questionnaire survey were processed using the statistical analysis method of independent t-test analysis, followed by the interpretation of the total score of each response item. Independent t-test analysis is used in data analysis by comparing two different pieces of data (Gravetter \& Wallnau, 2013). In this research, independent t-test analysis was used to determine whether gendered differences exist in the level of acceptance of children with special needs. This technique was intended to compare levels of acceptance amongst male and female respondents. The interpretation of the total score was based on the overall result of the number of scores in each response given by respondents in order to determine the level of acceptance of children with special needs.

A negative response (NO) scored 0, a neutral response (DON'T KNOW) scored 1, and a positive response (YES) scored 2. The higher the total score, the higher the level of acceptance, while the lower the total score, the lower the level of acceptance (Favazza \& Odom, 1996). The range of total scores in the ASK-R questionnaire is 0-36, classified into three levels of acceptance, where scores in the 0-11 range indicate low acceptance, 12-24 indicate moderate acceptance, and 25-36 indicate high acceptance (Nikolaraizi et al. 2005). However, based on the number of questions used in this research, the total score range was 0 28, with scores in the 0-8 range indicating low acceptance, 9-19 indicating moderate acceptance, and 20-28 indicating high acceptance. An independent t-test analysis was also conducted on gender data.

\section{Result}

The findings of the research suggest that levels of peer acceptance within the Inclusive Kindergarten are moderate. In this study, three categories were used to classify the level of peer acceptance: low, medium, and high. Descriptive statistical analysis revealed an average score of 16.90 and a standard deviation of 7.518, which places the overall score in the 10-19 range, indicating a medium level of peer acceptance of children with disabilities.

For the ASK-R item about having friends with special needs, $64.8 \%$ of participants responded that they do not have friends with special needs. With regard to experiences of playing and interacting with children with special needs, $51.4 \%$ respondents claimed no experience of playing with children with special needs, while $56.2 \%$ claim no experience interacting with children with special needs. However, children scored far higher in their desire and willingness to interact and play with children with special needs.

The research used independent t-test analysis to measure gender-based differences in levels of peer acceptance. The analysis suggests that peer acceptance was higher amongst boys $(\mathrm{M}=$ 17.81 with $\mathrm{SD}=7,021)$ compared to girls $(\mathrm{M}=16.00$ with $\mathrm{SD}=7,940)$ (Table 1$)$. However, based on the Sig value of .220 (>0.05), the difference is not significant, and it can be stated, therefore, that boys and girls exhibit the same level of peer acceptance. 
Table I. Mean of Total Score of ASK-R (Boys and Girls)

\begin{tabular}{lll}
\hline \multicolumn{1}{c}{ Gender } & Mean & $\begin{array}{l}\text { Standard } \\
\text { Deviation }\end{array}$ \\
\hline Boys & 17.81 & 7.021 \\
Girls & 16.00 & 7.940 \\
\hline
\end{tabular}

\section{Discussion}

This study aimed to describe the level of peer acceptance in early childhood in inclusive kindergarten education institutions for children with special needs. Based on the results described above, children who attend inclusive kindergartens were found to have moderate or neutral peer acceptance of children with special needs. Levels of acceptance showed no significant gender-based difference. The results of demographic factor analysis are in line with the most current research related to gender-based peer acceptance. Recent research has found no difference between boys and girls in their acceptance of children with special needs (Nikolaraizi et al., 2005; Hong, Kwon, \& Jeon, 2014). It should be noted, however, that other research has found that girls are more accepting than boys (de Boer, Pijl, \& Minnaert, 2012).

The results of this analysis of early childhood acceptance of children with special needs in inclusive education institutions found that most children are in the medium category or the neutral category. These results differ from previous research findings which indicate that children in inclusive education environments are more accepting of children with special needs (Nikolaraizi et al., 2005). Acceptance of children with special needs is expected to be higher in inclusive education settings because children with and without special needs can interact on a daily basis. Other studies have found that greater frequency of interaction has a positive influence on how accepting typically developing children are of their special needs peers (Hong, Kwon, \& Jeon, 2014).

Based on the results of the descriptive analysis conducted in the present study, it was found that children in inclusive early childhood education settings do not have sufficient experience interacting and playing with children with special needs. Also, despite being in an inclusive environment, most children do not have friends with special needs. This may be due to the very recent implementation of inclusive education in early childhood education in Indonesia, and children in these settings lack both experience interacting with children with special needs and information about these children. In addition to the experience of interaction, knowledge is an influencing factor in peer acceptance of children with special needs (de Boer, Pijl, \& Minnaert, 2012).

Acceptance by early years children in the medium category may arise from their limited cognitive development which means they do not view other children as having special needs and their limited knowledge of the concept of special needs. The research found that most early years children did not understand the words "disability" or "special needs." Instead, children identified differences between themselves and others based on visible physical conditions and the ability of other children to participate in play (Dyson, 2005) This finding is 
supported by other research that has shown that in early childhood, children prefer to play with peers who do not have disabilities rather than children with special needs. Children's selection and decision-making processes are influenced by several factors, including type of disability, form of activities that will take place, and other factors related to fairness and equity (Diamond \& Hong, 2010).

It is important that knowledge and information about children with special needs is provided to typically developing children early in their lives in order to increase their acceptance of children with special needs (Ostorosky, Mouzourou, Dorsey, Favazza, \& Leboeuf, 2015). Limited perceptions of children with special needs can interfere with their interactions with them (Nikolaraizi et al. 2005). Therefore, an early understanding of children with special needs can foster positive feelings and enable children to make decisions regarding the inclusion or exclusion of children with special needs in play and other activities (Hong, Kwon, \& Jeon, 2014).

The results of this study require further assessment. Observations of interactions and behaviors between typical children and children with special needs should be made within the school setting. This needs to be done because with early childhood respondents and was, thus, limited by the cognitive development of those respondents. The results of this and future research can form the basis for intervention programs to support peer acceptance of children with special needs by providing information and understanding to children about disabilities and children with special needs.

\section{Conclusion}

This research found moderate or neutral acceptance of children with special needs amongst children in an early childhood education setting. No significant differences between the levels of acceptance of boys and girls were observed. This moderate or neutral level of acceptance results from lack of knowledge of and experience with children with special needs, despite the inclusive educational environment.

Limitations to this study should be addressed in future research. The primary limitation was the age range of respondents and the small sample size. Future research can provide an overview of acceptance levels across a wider age range of children in early childhood. It can also expand on this research by observing how early childhood acceptance arises in activities with children with special needs in the school environment.

Knowledge about disability and about special needs groups should be provided in early childhood. This knowledge is essential to provide awareness of the existence of individuals with special needs and to foster positive acceptance of these individuals in early childhood. One way to achieve this is through the use of illustrated storybooks to introduce typically developing children to children with special needs. 


\section{References}

Beazidou, E., \& Botsoglou, K. (2016). Peer acceptance and frienship in early childhood: the conceptual distinctions between them. Early child development and care, 186, 1615-1631.

De Boer, A., Pijl, S. J., \& Minnaert, A. (2012). Students' attitudes towards peers with disabilities: A review of the literature. International Journal of Disability, Development and Education, 59, 379-392.

Diamond, K. E., \& Hong, S.Y. (2010). Young children's decisions to include peers with disabilities in play. Journal of Early Intervention, 32, 163-177.

Dyson, L. L. (2005). Kindergarten children's understanding of and attitudes toward people with disabilities. Topics in Early Childhood Special Education, 25, 95-105.

Favazza, P. C., \& Odom, S. L. (1996). Use of the acceptance scale to measure attitudes of kindergarten-age children. Journal of Early Intervention, 20, 232-248.

Favazza, P. C., Phillipsen, L., \& Kumar, P. (2000). Measuring and promoting acceptance of young children with disabilities. Exceptional Children, 66, 491-508.

Gravetter, F. J., \& Wallanau, L. B. (2013). Statistics for The Behavioral Sciences (9 $9^{\text {th }}$ ed.). Boston, MA: Wadsworth Cengage Learning.

Gravetter, F. J., \& Forzano, L. B. (2016). Research Methods for The Behavioral Sciences (5 ${ }^{\text {th }}$ ed.). Boston, MA: Cengage Learning.

Hong. S. Y., Kwon. K., \& Jeon. H. J. (2014). Children's attitudes towards peers with disabilities: Associations with personal and parental factors. Infant and Child Development, 23, 170-193.

Nikolaraizi, M., Kumar, P., Favazza, P., Sideridis, G., Koulousiou, D., \& Riall, A. (2005). A cross-cultural examination of typically developing children's attitudes toward individuals with special needs. International Journal of Disability, Development, and Education, 52, 101-119.

Odom, S. L., Zercher, C., Li, S., Marquart, J. M., Sandall, S., \& Brown, W. H. (2006). Social acceptance and rejection of preschool children with disabilities: A mixed-method analysis. Journal of Educational Psychology, 98, 807-823.

Ostorosky, M. M., Mouzourou, C., Dorsey, E. A., Favazza, P. C., \& Leboeuf, L. M. (2015). Pick a book, any book: Using children's books to support positive attitudes toward peers with disabilities. Young Exceptional Children, 18, 30-43.

Yu, S., Ostrosky, M. M., \& Fowler, S. A. (2012). Measuring young children's attitudes toward peers with disabilities: Highlights from the research. Early Childhood Special Education, 20, 1-11. 